\title{
-NOTES-
}

\section{SOME REMARKS ON THE MECHANICAL THEORY OF FLUID MIXTURES*}

\author{
By MORTON E. GURTIN (Carnegie-Mellon University)
}

1. Introduction. In a recent paper [1], Müller established a general thermodynamical theory of fluid mixtures based on Truesdell's equations of balance (see, e.g., [2, Secs. 159, 215, 243]) and the Coleman-Noll [3] interpretation of the Clausius-Duhem inequality. Müller's theory, which was the first of its type to allow density gradients to enter the constitutive relations, is in complete agreement with classical thermochemistry, in contrast to previous theories in which such a dependence was not allowed.

Here I show that the corresponding inviscid, purely mechanical theory has the following interesting property: the linearized equations of the theory are hyperbolic, but in the nonlinear theory if the constituent stresses depend on the density gradients, the equations in general are not hyperbolic. Thus, roughly speaking, infinitesmal disturbances in concentration travel with finite speed, but finite disturbances generally do not. In fact, I prove that acceleration waves of arbitrary amplitude and direction are possible only when the stresses are independent of the density gradients. ${ }^{1}$ (See also [6].)

A theory similar to the one studied here was developed by Green and Adkins [4]; however, in their theory density gradients were omitted from the outset. ${ }^{2}$

2. Basic equations. We consider a mixture of $N$ constituents $(\alpha=1, \cdots, N)$; in the absence of chemical reactions the balance laws for mass and linear momentum take the form (see, e.g., [2, Secs. 159, 215]):

$$
\begin{gathered}
\rho_{\alpha}^{\prime}+\operatorname{div}\left(\rho_{\alpha} \nabla_{\alpha}\right)=0, \\
\rho_{\alpha} \nabla_{\alpha}^{\prime}+\rho_{\alpha}\left(\operatorname{grad} \nabla_{\alpha}\right) \nabla_{\alpha}=\operatorname{div} T_{\alpha}+1_{\alpha}+b_{\alpha} .
\end{gathered}
$$

Here $\rho_{\alpha}$ is the mass density of constituent $\alpha, \nabla_{\alpha}$ is the velocity of $\alpha, T_{\alpha}$ is the stress tensor for $\alpha, 1_{\alpha}$ is the momentum supply for $\alpha$, and $b_{\alpha}$ is the body force on $\alpha$. The total momentum supplied to the mixture must vanish; thus we add the requirement that (see, e.g., [2, Eq. (215.5)]):

$$
\sum 1_{\alpha}=0 .
$$

The total mass density $\rho$, the concentration $c_{\alpha}$ of $\alpha$, the velocity $\mathbf{v}$ of the mixture, and

* Received June 20, 1971.

${ }^{1}$ Bowen informs me that in his theory of fluid mixtures the stress is independent of the density gradients when the diffusion velocities vanish. Thus acceleration waves can exist in that theory when the region ahead of the wave is not undergoing diffusion. This, of course, does not contradict the results given here.

2 See also Bowen and Doria [7], who study the effects of diffusion on acceleration waves in gases. In their theory density gradients are not present in the constitutive equations.

3 Here and in what follows a prime denotes the spatial time derivative, grad and div denote the spatial gradient and spatial divergence, and $\Delta$ denotes the spatial Laplacian. 
the diffusion velocity $\mathbf{u}_{\alpha}$ of $\alpha$ are defined by

$$
\rho=\sum \rho_{\alpha}, \quad c_{\alpha}=\rho_{\alpha} / \rho, \quad \mathbf{v}=\sum c_{\alpha} \mathbf{v}_{\alpha}, \quad \mathbf{u}_{\alpha}=\mathbf{v}_{\alpha}-\mathbf{v} .
$$

We consider fluid mixtures defined by constitutive relations of the form (cf. [1, Eq. (4.2)]):

$$
\mathrm{T}_{\alpha}=\mathrm{T}_{\alpha}(\vec{\rho}, \operatorname{grad} \vec{\rho}, \overrightarrow{\mathfrak{u}}), \quad 1_{\alpha}=1_{\alpha}(\vec{\rho}, \operatorname{grad} \vec{\rho}, \overrightarrow{\mathfrak{u}}),
$$

where

$$
\vec{\rho}=\left(\rho_{1}, \cdots, \rho_{N}\right), \quad \operatorname{grad} \vec{\rho}=\left(\operatorname{grad} \rho_{1}, \cdots, \operatorname{grad} \rho_{N}\right), \quad \overrightarrow{\mathbf{u}}=\left(\mathbf{u}_{1}, \cdots, \mathbf{u}_{N}\right) .
$$

Further, since the constituents are fluids, we assume that $T_{\alpha}$ and $l_{\alpha}$ are isotropic functions.

3. Wave propagation in the linearized theory. Let $\dot{\rho}_{1}, \cdots, \dot{\rho}_{N}$ be given constant values of the constituent densities, and suppose that the following quantities are small: $\left|\rho_{\alpha}-\dot{\rho}_{\alpha}\right|,\left|\rho_{\alpha}^{\prime}\right|,\left|\operatorname{grad} \rho_{\alpha}\right|,\left|\nabla_{\alpha}\right|,\left|v_{\alpha}^{\prime}\right|,\left|\operatorname{grad} \nabla_{\alpha}\right|$. Then "to within second-order terms" the constitutive relations (4) reduce to

$$
\begin{aligned}
\mathrm{T}_{\alpha} & =-p_{\alpha} 1, \quad p_{\alpha}=\sum_{\beta} A_{\alpha \beta}\left(\rho_{\beta}-\dot{\rho}_{\beta}\right), \\
1_{\alpha} & =-\sum_{\beta}\left\{C_{\alpha \beta} \operatorname{grad} \rho_{\beta}+D_{\alpha \beta} \mathrm{u}_{\beta}\right\},
\end{aligned}
$$

where $A_{\alpha \beta}, C_{\alpha \beta}$, and $D_{\alpha \beta}$ are constants and

$$
\sum_{\alpha} C_{\alpha f}=\sum_{\alpha} D_{\alpha \ell}=0 .
$$

The derivation of (5) and (6) makes use of (2) and the assumed isotropy of the material. Further, to the same degree of approximation the field equations (1) have the form

$$
\rho_{\alpha}^{\prime}+\dot{\rho}_{\alpha} \operatorname{div} \nabla_{\alpha}=0, \quad \dot{\rho}_{\alpha} \nabla_{\alpha}^{\prime}=-\operatorname{grad} p_{\alpha}+1_{\alpha}+\mathrm{b}_{\alpha},
$$

while

$$
\mathrm{v}=\sum \dot{c}_{\alpha} \nabla_{\alpha}, \quad \dot{c}_{\alpha}=\dot{\rho}_{\alpha} / \dot{\rho}, \quad \dot{\rho}=\sum \dot{\rho}_{\alpha} .
$$

Eqs. (5) and (7), supplemented by $(3)_{4},(6)$ and (8), constitute the basic field equations of the linearized theory.

By (7) ${ }_{1}$ and (8), $\rho^{\prime}+\dot{\rho} \operatorname{div} v=0$, and this result, $(3)_{4},(7)_{1}$, and $(8)_{2}$ imply

$$
\dot{\rho}_{\alpha} \operatorname{div} \mathbf{u}_{\alpha}=-\rho_{\alpha}^{\prime}+\dot{c}_{\alpha} \rho^{\prime} .
$$

If we take the time derivative of $(7)_{1}$ and the divergence of $(7)_{2}$, we conclude, with the aid of (5) 2,3 and (9), that (cf. [4, Eq. (6.4)])

$$
\rho_{\alpha}^{\prime \prime}=\dot{\rho}_{\alpha} \sum_{\beta} L_{\alpha \beta} \Delta_{\rho_{\beta}}-\sum_{\beta} \frac{D_{\alpha \beta}}{\dot{\rho}_{\beta}}\left(\rho_{\beta}^{\prime}-\dot{c}_{\beta} \rho^{\prime}\right)-\operatorname{div} \mathrm{b}_{\alpha},
$$

where

$$
L_{\alpha \beta}=\frac{1}{\dot{\rho}_{\alpha}}\left[A_{\alpha \beta}+C_{\alpha \beta}\right] .
$$

If $\left\|L_{\alpha \beta}\right\|$ is symmetric ${ }^{4}$ and positive definite, then (10) is hyperbolic and hence admits 1 Gurtin [5, Corollary 5.1] has shown that the symmetry of $\left\|L_{\alpha \beta}\right\|$ is a consequence of the second law. 
discontinuous solutions of all orders. Moreover, if $a_{\alpha}$ is the amplitude and $U$ the speed of propagation of such a disturbance, then

$$
\sum_{\beta} L_{\alpha \beta} a_{\beta}=\frac{1}{\dot{\rho}_{\alpha}} U^{2} a_{\alpha} .
$$

We shall show in the next section that, in contrast to the linear theory, discontinuous solutions are in general not possible in the nonlinear theory.

It is of interest to note that, by $(5)_{3}$ and (11), $1_{\alpha}$ is independent of $\operatorname{grad} \vec{\rho}$ (in the linear theory) if and only if

$$
L_{\alpha \beta}=\frac{1}{\dot{\rho}_{\alpha}} A_{\alpha \beta} .
$$

It may be possible to determine $A_{\alpha \beta}$ without using the results of wave propagation experiments. If this is done, and if the propagation speeds are measured, then (12) can be used to determine whether or not (13) holds, and hence whether or not $1_{\alpha}$ depends on $\operatorname{grad} \vec{\rho}$.

4. Wave propagation in the nonlinear theory. Consider the system of partial differential equations (1), (4) for $\rho_{\alpha}$ and $\nabla_{\alpha}$. If $\mathbf{T}_{\alpha}$ depends on grad $\vec{\rho}$, then the term $\operatorname{div} \mathbf{T}_{\alpha}$ will involve $\operatorname{grad}^{2} \vec{\rho}$; thus this system of equations will in general be nonhyperbolic. This is in contrast to the linear theory in which the equations are hyperbolic. Of course, as is clear from $(5)_{1,2}$, the stress in the linear theory is independent of grad $\vec{\rho}$.

From the discussion given in the preceding paragraph one would expect that acceleration waves of arbitrary amplitude and direction are not possible when $\mathbf{T}_{\alpha}$ depends on $\operatorname{grad} \vec{\rho}$. We now show that this conjecture is correct. An acceleration wave for the mixture is defined exactly as for a single body. Thus across such a wave [2, Sec. 190]:

$$
\vec{\rho} \text { and } \overrightarrow{\mathbf{u}} \text { are continuous, }
$$

grad $\vec{\rho}$ suffers a jump discontinuity.

In addition, if $\mathbf{n}$ denotes the direction of propagation, we also have the dynamical condition of compatibility [2, Eq. (205.5)]:

$$
\left[\mathrm{T}_{\alpha}\right] \mathbf{n}=\mathbf{0},
$$

where $\left[\mathbf{T}_{\alpha}\right]$ denotes the jump in $\mathbf{T}_{\alpha}$ across the wave. We define the amplitude of the wave (at a given point) to be the array $\left\{\vec{\rho}, \overrightarrow{\mathrm{u}},(\operatorname{grad} \vec{\rho})^{-},(\operatorname{grad} \vec{\rho})^{+}\right\}$, where $\rho_{\alpha}$ and $\mathbf{u}_{\alpha}$ denote the values of the density and diffusion velocity of $\alpha$ at the wave, while (grad $\left.\rho_{\alpha}\right)^{-}$ and $\left(\operatorname{grad} \rho_{\alpha}\right)^{+}$denote the values of the density gradient for $\alpha$ immediately behind and just in front of the wave. By (4) ${ }_{1}$ and (14),

$$
\mathbf{T}_{\alpha}\left(\rho,(\operatorname{grad} \vec{\rho})^{-}, \overrightarrow{\mathbf{u}}\right) \mathbf{n}=\mathbf{T}_{\alpha}\left(\vec{\rho},(\operatorname{grad} \vec{\rho})^{+}, \overrightarrow{\mathbf{u}}\right) \mathbf{n},
$$

and this relation implies the following

TheOREM: If (at a given point in the mixture) an acceleration wave of arbitrary amplitude and direction is possible, then the stress for each constituent must be independent of the density gradient; i.e., (4) $)_{1}$ must reduce to

$$
\mathbf{T}_{\alpha}=\mathbf{T}_{\alpha}(\vec{\rho}, \overrightarrow{\mathrm{u}}) .
$$

I conjecture that the presence of grad $\vec{\rho}$ leads to "structured waves" whose thickness is of the order of $|\operatorname{grad} \vec{\rho}|$. 


\section{References}

[1] I. Müller, A thermodynamic theory of mixtures of fluids, Arch. Rational Mech. Anal. 28, 1-39 (1968)

[2] C. Truesdell and R. A. Toupin, The classical field theories, Encyclopedia of Physics III/1, Springer, Berlin-Göttingen-Heidelberg, 1960

[3] B. D. Coleman and W. Noll, The thermodynamics of elastic materials with heat conduction and viscosity, Arch. Rational Mech. Anal. 13, 167-178 (1963)

[4] A. E. Green and J. E. Adkins, A contribution to the theory of non-linear diffusion, Arch. Rational Mech. Anal. 15, 235-246 (1964)

[5] M. E., Gurtin, On the thermodynamics of chemically reacting fluid mixtures, Arch. Rational Mech. Anal. 43, 198-212 (1971)

[6] A. E. Green and P. M. Naghdi, $A$ note on mixtures, Int. J. Engng. Sci. 6, 631-635 (1968)

[7] R. M. Bowen and M. L. Doria, On the effect of diffusion on the growth and decay of acceleration waves in gases (to appear in J. Acoust. Soc. Am.) 